\title{
Specific organ metastases and survival in small cell lung cancer
}

\author{
KENSUKE NAKAZAWA $^{1}$, KOICHI KURISHIMA ${ }^{1}$, TOMOHIRO TAMURA $^{1}$, KATSUNORI KAGOHASHI $^{2}$, \\ HIROICHI ISHIKAWA ${ }^{3}$, HIROAKI SATOH ${ }^{2}$ and NOBUYUKI HIZAWA ${ }^{1}$ \\ ${ }^{1}$ Division of Respiratory Medicine, Institute of Clinical Medicine, University of Tsukuba, Mito, Ibaraki 305-8575; \\ ${ }^{2}$ Division of Respiratory Medicine, Mito Medical Center, University of Tsukuba, Mito, Ibaraki 310-0015; \\ ${ }^{3}$ Division of Respiratory Medicine, Tsukuba Medical Center Hospital, Tsukuba, Ibaraki 305-8558, Japan
}

Received March 15, 2012; Accepted June 26, 2012

DOI: 10.3892/ol.2012.792

\begin{abstract}
The aim of our retrospective study was to evaluate the clinicopathological features associated with distant metastasis from small cell lung cancer (SCLC). We reviewed patients diagnosed with SCLC metastasis at the time of presentation between 1999 and 2010. Among the consecutive 251 SCLC patients diagnosed, $152(60.6 \%)$ patients had distant metastasis, of which 20.3, 18.3, 15.5, 10.0 and $6.0 \%$ of patients had liver, bone, brain, lung and adrenal gland metastasis, respectively. In a multivariate analysis using Cox's proportional hazards model, we identified that liver, bone and brain metastasis as well as the presence of pleural and/ or pericardial fluids were unfavorable prognostic factors. However, lung, adrenal gland and extrathoracic lymph node metastasis were not statistically significant prognostic factors. With regard to the treatment of SCLC patients, particularly those with liver, bone and brain metastasis or pleural and/or pericardial fluids, we should take the metastasizing organs into consideration.
\end{abstract}

\section{Introduction}

Distant metastases upon presentation of small cell lung cancer (SCLC) is a frequent clinical problem. Clinically, treatment for extensive disease (ED)-SCLC consists of systemic chemotherapy and radiotherapy for symptomatic metastatic sites. It is generally accepted that the life expectancy of SCLC patients depends on the extent of disease and the response to chemotherapy. Performance status (PS), gender, disease extent and response to chemotherapy have been evaluated as prognostic factors (1). Foster et al revealed that the number of metastatic sites was also a prognostic factor in ED-SCLC

Correspondence to: Professor Hiroaki Satoh, Division of Respiratory Medicine, Mito Medical Center, University of Tsukuba, Miya-machi 3-2-7, Mito, Ibaraki 310-0015, Japan

E-mail: hirosato@md.tsukuba.ac.jp

Key words: small cell lung cancer, metastasis, survival patients, regardless of the location of the metastatic site (2). The four most common sites of metastasis in SCLC at the time of diagnosis appear to be the liver, bone, brain and lung, with involvement of these sites identified in SCLC patients with newly diagnosed metastatic disease. Using the database of SCLC patients, we examined whether specific organ metastasis at the time of presentation had prognostic significance in SCLC patients.

\section{Patients and methods}

Patients. We retrospectively analyzed 251 patients who were diagnosed with SCLC in our divisions at the University of Tsukuba and the Tsukuba Medical Center Hospital between January 1999 and May 2010. This retrospective study conformed to the Ethical Guidelines for Clinical Studies issued by the Ministry of Health, Labor and Welfare of Japan. Analysis of the medical records of the lung cancer patients was approved by the ethics committee of the University of Tsukuba Hospital, Ibaraki, Japan. The diagnosis of SCLC was confirmed in all patients using pathological and/or cytological specimens. Pathological diagnosis of SCLC was defined by the WHO classification (3). Prior to treatments, a staging procedure was conducted for all patients using computed tomography (CT) or magnetic resonance imaging (MRI) of the head, bone scintigraphy and ultrasonography and/or CT of the abdomen. Patients were staged according to the staging system of the Veterans Administration Lung Cancer Study Group into one of two categories; limited-stage disease (LD) or extensive-stage disease (ED) (4,5). Patients with LD-SCLC had metastatic disease restricted to the ipsilateral hemithorax within a single radiation port, while patients with ED-SCLC had widespread metastatic disease $(4,5)$.

Statistical analysis. The statistical significance between the two groups was determined using the Mann-Whitney U test and the Chi-square test. Logistic regression analysis was applied in order to examine the significance of the seven variables, including liver, bone, brain, lung, extrathoracic lymph node and adrenal metastasis as well as pleural and/ or pericardial fluid, for poor PS and unfavorable response to chemotherapy. The Kaplan-Meier method was used to 
assess the survival curves and the log-rank test was used to evaluate the statistically significant differences between the two groups. The length of survival was defined in months as the interval from the date of initial therapy or supportive care until the date of mortality or last follow-up. Cox's proportional hazards model was used to study the effects of clinicopathological factors on survival (6). All statistical analyses were performed using SPSS version 10.1 for Windows (SPSS, Chicago, IL, USA) and $\mathrm{P}<0.05$ was considered to indicate a statistically significant difference.

\section{Results}

Patient characteristics. The diagnosis of all 251 SCLC patients was pathologically and/or cytologically confirmed. The characteristics of these patients are shown in Table I. Among the 251 SCLC patients, 222 (88.4\%) were male and 29 were female. The mean age was 71 years (range, $41-86$ years). A total of $73(29.1 \%)$ patients had pleural and/or pericardial fluids and among the $152(60.6 \%)$ patients that had distant metastasis, 51 (20.3\%), 46 (18.3\%), 39 (15.5\%), 25 (10.0\%) and $15(6.0 \%)$ patients also had liver, bone, brain, lung and adrenal gland metastasis, respectively.

Number of metastatic sites infuences survival rate. A total of 69 patients had one metastatic site, 28 had two, 20 had three, four had four and one had five (data not shown). A statistically significant difference was observed in the survival rate among patients without metastasis, those with one metastatic site and those with two or more metastatic sites $(\mathrm{P}=0.0001)$ (Fig. 1).

A total of 16 patients had sole liver metastasis, while 35 patients had liver metastasis accompanied by metastasis in another site. Additionally, 12, 22, and six patients had sole bone, brain, and lung metastasis, respectively. A total of 34, 17 and 19 patients had bone, brain and lung metastasis accompanied by metastasis in another site, respectively. A statistically significant difference was observed in the one-year survival between the patients with sole liver metastasis and those without metastasis $(\mathrm{P}=0.0009)$. Similar results were observed between those with sole bone $(\mathrm{P}=0.0013)$, brain $(\mathrm{P}=0.0163)$ and lung $(\mathrm{P}=0.169)$ metastasis, and those without metastasis, respectively (Table II).

Metastasis affects response to chemotherapy. According to logistic regression analysis, liver metastasis, brain metastasis and pleural and/or pericardial fluid are correlated with a poor PS of 2-3 (Table III). A logistic regression analysis also demonstrated that liver and brain metastasis are risk factors of unfavorable response to chemotherapy (stable disease and progressive disease; Table IV).

Multivariate analysis. In a multivariate analysis using Cox's proportional hazards model, presence of liver metastasis $(\mathrm{P}=0.0001)$, bone metastasis $(\mathrm{P}=0.0401)$, brain metastasis $(\mathrm{P}=0.0177)$ and pleural and/or pericardial fluids $(\mathrm{P}=0.0020)$ were unfavorable prognostic factors. However, lung $(\mathrm{P}=0.7028)$, adrenal gland $(\mathrm{P}=0.2405)$ and extrathoracic lymph node metastasis $(\mathrm{P}=0.0672)$ were not statistically significant prognostic factors (Table V).
Table I. Characteristics of 251 patients with SCLC.

Patient characteristics

No. of patients $(\%)$

$\begin{array}{lc}\text { Age (years) } & \\ \text { Median } & 71 \\ \text { Range } & 41-86 \\ \text { Gender } & \\ \text { Male } & 222(88.4) \\ \text { Female } & 29(11.6) \\ \text { Distant metastasis } & \\ \text { Present } & 152(60.6) \\ \text { Absent } & 99(39.4) \\ \text { Clinical stage } & \\ \text { Limited } & 78 \\ \text { Extensive } & 125 \\ \text { Metastatic site } & \\ \text { Pleural and/or pericardial fluids } & 73(29.1) \\ \text { Liver } & 51(20.3) \\ \text { Bone } & 46(18.3) \\ \text { Brain } & 39(15.5) \\ \text { Lung } & 25(10.0) \\ \text { Adrenal gland } & 15(6.0) \\ \text { Extrathoracic lymph node } & 15(6.0) \\ \text { Pancreas } & 3(1.2) \\ \end{array}$

SCLC, small cell lung cancer.

Table II. Metastatic sites and comparison of the one-year survival between patients with sole specific organ metastasis and patients without metastasis.

\begin{tabular}{lccc}
\hline $\begin{array}{l}\text { Metastatic } \\
\text { site }\end{array}$ & $\begin{array}{c}\text { One-year } \\
\text { survival (\%) }\end{array}$ & $\begin{array}{l}\text { No. of patients with } \\
\text { sole metastatic sites }\end{array}$ & P-value \\
\hline None & 54.3 & & \\
Liver & 18.8 & 16 & 0.0009 \\
Bone & 25.0 & 12 & 0.0013 \\
Brain & 40.9 & 22 & 0.0163 \\
Lung & 33.4 & 6 & 0.0169 \\
\hline
\end{tabular}

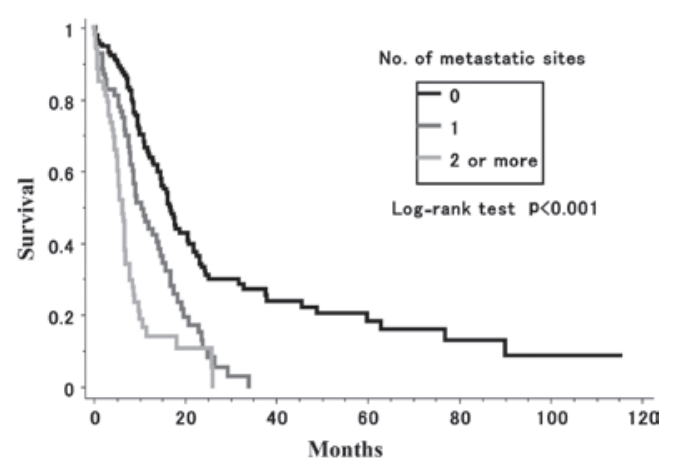

Figure 1. Comparison of survival between patients without metastasis, patients with one metastatic site and patients with two or more metastatic sites. 
Table III. Logistic regression analysis for the factors of a poor PS (PS 2-3).

\begin{tabular}{lccc}
\hline Independent factor & Hazard ratio & $95 \%$ CI & P-value \\
\hline Liver & 2.311 & $1.107-4.823$ & 0.0256 \\
Bone & 1.505 & $0.683-3.313$ & 0.3103 \\
Brain & 2.634 & $1.244-5.579$ & 0.0114 \\
Lung & 0.819 & $0.310-2.164$ & 0.6887 \\
Adrenal gland & 1.330 & $0.401-4.409$ & 0.6404 \\
Extra-thoracic lymph node & 1.096 & $0.355-3.377$ & 0.8737 \\
Pleural and/or pericardial fluid & 3.066 & $1.660-5.663$ & 0.0003 \\
\hline
\end{tabular}

PS, performance status; CI, confidence interval.

Table IV. Logistic regression analysis for the factors of an unfavorable response to chemotherapy (stable disease and progressive disease).

\begin{tabular}{lcll}
\hline Independent factor & Hazard ratio & 95\% CI & P-value \\
\hline Liver & 5.304 & $2.201-12.785$ & 0.0002 \\
Bone & 0.528 & $0.178-1.563$ & 0.2484 \\
Brain & 2.695 & $1.073-6.769$ & 0.0348 \\
Lung & 1.571 & $0.508-4.856$ & 0.4329 \\
Adrenal gland & 2.885 & $0.734-11.388$ & 0.1291 \\
Extrathoracic lymph node & 1.339 & $0.369-4.859$ & 0.6568 \\
Pleural and/or pericardial fluid & 1.535 & $0.721-3.270$ & 0.2664 \\
\hline
\end{tabular}

CI, confidence interval.

Table V. Multivariate analysis of prognostic factors in patients with SCLC.

\begin{tabular}{lccr}
\hline & \multicolumn{3}{c}{ Multivariate analysis (Cox's proportional hazards model) } \\
\cline { 2 - 4 } Factor & Hazard ratio & $95 \%$ CI & P-value \\
\hline Liver & 0.414 & $0.273-0.627$ & 0.0001 \\
Bone & 0.643 & $0.422-0.980$ & 0.0401 \\
Brain & 0.608 & $0.403-0.917$ & 0.0177 \\
Lung & 0.903 & $0.534-1.527$ & 0.7028 \\
Adrenal gland & 0.689 & $0.369-1.284$ & 0.2405 \\
Extrathoracic lymph node & 0.586 & $0.331-1.039$ & 0.0672 \\
Pleural and/or pericardial fluid & 0.608 & $0.443-0.833$ & 0.0020
\end{tabular}

SCLC, small cell lung cancer; CI, confidence interval.

\section{Discussion}

The incidence of distant metastasis at the time of initial diagnosis of SCLC in this study was $60.6 \%$, and the most common metastatic sites were the liver, bone, brain, lung and adrenal glands. In this study, we examined whether specific organ metastasis at the time of presentation had prognostic significance in SCLC patients. For decades, the prognostic significance of bone marrow involvement in SCLC patients has been reported $(7,8)$. Thereafter, several studies indicated that patients with multiple metastatic sites had a significantly poor survival rate (9-11). Our results revealed that there was a significant difference in survival among patients without metastasis compared to those with one metastatic site and those with two or more metastatic sites. These results were consistent with previous studies (9-11). In these studies, the site of involvement did not appear to have an impact on survival $(2,9)$; however, other researchers have identified that specific organ metastasis is related to poor prognosis (12-14). Bremnes et al reported that liver and brain metastasis were independent prognostic 
factors in ED-SCLC (12). Mohan et al also revealed that brain metastasis was a significant predictor of survival (13). In a study of 116 SCLC patients, Arinc et al indicated that bone metastasis was one of the significant prognostic factors in univariate analysis, but was not identified as a prognostic factor in multivariate analysis (14). In the present study, univariate analysis revealed that patients with sole organ metastasis in the liver, bone, brain and lung had a poorer prognosis compared to those without metastasis. However, in multivariate analysis, liver, bone and brain metastasis were unfavorable prognostic factors, while lung metastasis was not. In our logistic regression analysis, the presence of liver metastasis, brain metastasis and pleural and/or pericardial fluid demonstrated a statistically significant correlation with poor PS. Liver metastasis and brain metastasis also correlated with an unfavorable response to chemotherapy. Therefore, these results indicate that liver metastasis, brain metastasis and pleural and/or pericardial fluid have certain associations with poor prognosis.

Nussbaum et al identified that multiple brain metastases were common in patients with SCLC (15). Activity of daily living (ADL) may be low in a number of patients with brain metastases, and may also deteriorate in patients with bone metastases due to pain and fracture. The discontinuation of chemotherapy due to the deterioration of ADL in these patients has a certain correlation with poor survival. With regard to liver metastasis, the majority of SCLC patients had multiple nodules in our previous study (16). SCLC may cause biliary tract obstruction by metastasizing to lymph nodes in the porta hepatis or hepatic parenchyma $(17,18)$. The administration of chemotherapy may be complicated by metastasis of the liver in the activation or metabolism of several cytotoxic drugs commonly used in the treatment of SCLC. Lung metastatic lesions usually respond well to chemotherapy, and these lesions rarely cause complications unless hemoptysis and respiratory tract obstruction develop. Further studies are required to confirm the impact of metastasis of these organs as observed in the present study.

Recently, Ignatius Ou and Zell reported the applicability of the proposed International Association for the Study of Lung Cancer (IASLC) staging for SCLC, in comparison with the current Union for International Cancer Control (UICC) 6th Tumor Node Metastasis (TNM) edition (19). They concluded that patients with pleural and/or pericardial effusion had a poorer prognosis compared to those with metastatic disease (19). In the UICC TNM 7th edition, patients with pleural effusion have an intermediate prognosis between LD- and EL-SCLC (20). Our present study also confirmed that patients with pleural and/or pericardial effusion had a poorer prognosis compared to those without.

Despite these significant findings, this study has limitations. Firstly, the retrospective design of the study meant that it was complicated by lead time and length time biases. Secondly, there was a lack of information with regard to the development of distant metastases in the patients' clinical courses. Regardless of these limitations, we recognize that our findings have certain clinical importance for the management of future SCLC patients of unselected groups.

In conclusion, the therapeutic approach for treating SCLC patients with distant metastases is complicated, as our results suggest that existing liver, bone and brain metastasis adversely affects the outcome of the disease. When deciding whether or not to offer an intensive therapy, which may increase treatment-related mortality, the patient's medical condition, including coexistence of such metastases, should be taken into consideration, although favorable results have been reported in a number of these patients.

\section{References}

1. Ferraldeschi R, Baka S, Jyoti B, Faivre-Finn C, Thatcher N and Lorigan P: Modern management of small-cell lung cancer. Drugs 67: 2135-2152, 2007.

2. Foster NR, Mandrekar SJ, Schild SE, et al: Prognostic factors differ by tumor stage for small cell lung cancer: a pooled analysis of North Central Cancer Treatment Group trials. Cancer 115: 2721-2731, 2009.

3. Travis W, Brambilla E, Muller-Hermelink H and Harris C (eds): Tumours of the lung, pleura, thymus and heart. In: Pathology and Genetics World Health Organisation Classification of Tumours. IARC Press, Lyon, 2004.

4. Zelen M: Keynote address on biostatistics and data retrieval. Cancer Chemother Rep 3 4: 31-42, 1973.

5. Shepherd FA, Ginsberg RJ, Haddad R, et al: Importance of clinical staging in limited small-cell lung cancer: a valuable system to separate prognostic subgroups. The University of Toronto Lung Oncology Group. J Clin Oncol 11: 1592-1597, 1993.

6. Cox DR: Regression models and life tables. J Roy Statist Soc Ser B Metho 34: 187-220, 1972.

7. Bezwoda WR, Lewis D and Livini N: Bone marrow involvement in anaplastic small cell lung cancer. Diagnosis, hematologic features, and prognostic implications. Cancer 58: 1762-1765, 1986.

8. Zych J, Polowiec Z, Wiatr E, Broniek A and Rowinska-Zakrzewska E: The prognostic significance of bone marrow metastases in small cell lung cancer patients. Lung Cancer 10: 239-245, 1993.

9. Tas F, Aydiner A, Topuz E, Camlica H, Saip P and Eralp Y: Factors influencing the distribution of metastases and survival in extensive disease small cell lung cancer. Acta Oncol 38: 1011-1015, 1999.

10. Li J, Dai CH, Chen P, et al: Survival and prognostic factors in small cell lung cancer. Med Oncol 27: 73-81, 2010.

11. Tamura M, Ueoka H, Kiura K, et al: Prognostic factors of small-cell lung cancer in Okayama Lung Cancer Study Group Trials. Acta Med Okayama 52: 105-111, 1998.

12. Bremnes RM, Sundstrom S, Aaseb $\varnothing$ U, Kaasa S, Hatlevoll R and Aamdal S: Norweigian Lung Cancer Study Group: The value of prognostic factors in small cell lung cancer: results from a randomised multicenter study with minimum 5 year follow-up. Lung Cancer 39: 303-313, 2003.

13. Mohan A, Goyal A, Singh P, et al: Survival in small cell lung cancer in India: prognostic utility of clinical features, laboratory parameters and response to treatment. Indian J Cancer 43: 67-74, 2006.

14. Arinc $\mathrm{S}$, Gonlugur U, Devran O, et al: Prognostic factors in patients with small cell lung carcinoma. Med Oncol 27: 237-241, 2010.

15. Nussbaum ES, Djalilian HR, Cho KH and Hall WA: Brain metastases. Histology, multiplicity, surgery, and survival. Cancer 78: 1781-1988, 1996.

16. Kagohashi K, Satoh H, Ishikawa H, Ohtsuka M and Sekizawa K: Liver metastasis at the time of initial diagnosis of lung cancer. Med Oncol 20: 25-28, 2003.

17. Obara M, Satoh H, Yamashita YT, et al: Metastatic small cell lung cancer causing biliary obstruction. Med Oncol 15: 292-294, 1998

18. Ogawa R, Kodama T, Kurishima K, Kagohashi K and Satoh H: Portal vein tumor thrombus of liver metastasis from lung cancer. Acta Medica (Hradec Kralove) 52: 163-166, 2009.

19. Ignatius Ou SH and Zell JA: The applicability of the proposed IASLC staging revisions to small cell lung cancer (SCLC) with comparison to the current UICC 6th TNM Edition. J Thorac Oncol 4: 300-310, 2009.

20. Shepherd FA, Crowley J, Van Houtte P, et al: The International Association for the Study of Lung Cancer lung cancer staging project: proposals regarding the clinical staging of small cell lung cancer in the forthcoming (seventh) edition of the tumor, node, metastasis classification for lung cancer. J Thorac Oncol 2: 1067-1077, 2007 